\title{
Lexicalization of natural actions and cross-linguistic stability
}

Paul E. Hemeren ${ }^{1}$, Sofia Kasviki ${ }^{1}$ and Barbara Gawronska ${ }^{2}$

${ }^{1}$ School of Humanities and Informatics, University of Skövde, Sweden

${ }^{2}$ Department of Foreign Languages and Translation, University of Agder, Norway

https://doi.org/10.36505/ExLing-2008/02/0027/000086

\begin{abstract}
To what extent do Modern Greek, Polish, Swedish and American English similarly lexicalize action concepts, and how similar are the semantic associations between verbs denoting natural actions? Previous results indicate cross-linguistic stability between American English, Swedish, and Polish in verbs denoting basic human body movement, mouth movements, and sound production. The research reported here extends the cross-linguistic comparison to include Greek, which, unlike Polish, American English and Swedish, is a path-language. We used action imagery criteria to obtain lists of verbs from native Greek speakers. The data were analyzed by using multidimensional scaling, and the results were compared to those previously obtained.
\end{abstract}

Key words: motion verbs, natural actions, cross-linguistic stability, manner, path

\section{Introduction}

Human actions represent a conceptual domain where motion plays an important role in category structure and lexicalization as well as recognition. Learning to perceptually discriminate among various actions requires access to the spatiotemporal patterns in human movement (Giese and Poggio 2003). It seems that the reverse is also true: retrieving natural actions from memory requires access to spatiotemporal features associated with specific actions.

If perceptual criteria play a fundamental role in the lexicalization of action concepts, then we should see some cross-linguistic stability in mental lexical representations despite the fact that previous research (Vinson and Vigliocco 2002) has shown that the semantic space for verbs contains less well-defined boundaries in contrast to the semantic space for nouns.

In previous research, Hemeren (1996) and Hemeren and Gawronska (2007) asked American, Polish, and Swedish informants to spontaneously list verbs denoting actions that can be easily recognized when seen and can be visualized as a mental image. The results showed a clear tendency for informants to list verbs for basic level actions first. Verbs for subordinate level actions were mentioned later and less frequently. In all three languages investigated, we found significant correlations between total verb frequency (TF) and the mean ordinal position (MOP). By using multidimensional scaling as a technique to investigate the structure of semantic associations

ExLing 2008: Proceedings of 2nd Tutorial and Research Workshop on Experimental Linguistics, 25-27 August 2008, Athens, Greece 
within a language, further analyses revealed a similar tendency for the three languages: basic motion verbs 'run', walk', jump' and verbs referring to mouth motion and/or sound production: 'laugh', 'talk', 'sing' had a similar organization in terms of derived distances in the three-dimensional semantic space. These results indicate a tendency towards cross-linguistic stability between motion verbs and to a certain extent the mouth motion/vocal verbs.

\section{The current study}

The research presented here compares data coming from speakers of "manner languages", like English, Swedish, and Polish, where the manner of motion is normally encoded in the semantics of the main verb, and from speakers of "path languages", like Greek.

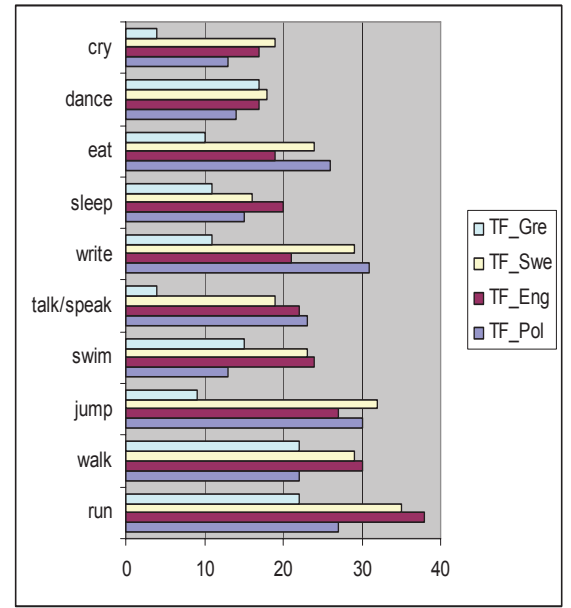

A

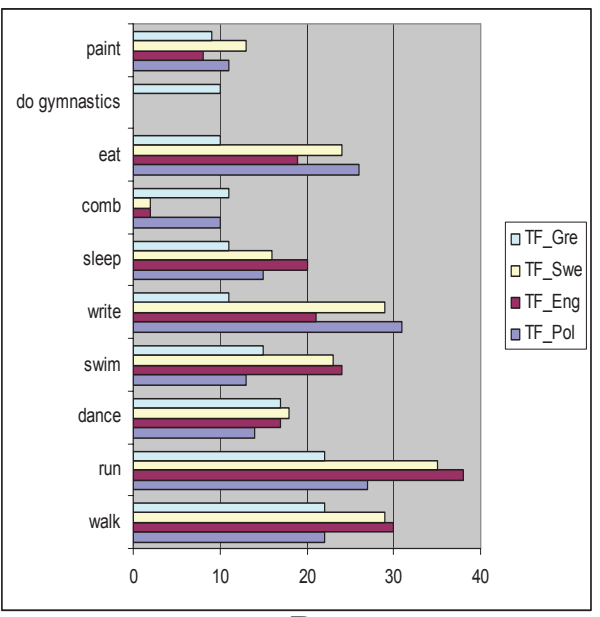

B

Figure 1. Panel A shows the 10 most frequent English verbs compared with the frequencies of the corresponding verbs in Greek, Polish, and Swedish. Panel B shows the 10 most frequent Greek verbs compared with the frequencies of the corresponding verbs in English, Polish, and Swedish.

We applied the same method as in the previous investigations. Thirty-five native speakers of Greek were asked to spontaneously list verbs denoting actions that are easy to imagine. The data were first analyzed with respect to verb frequency, list position, and the correlation between those two measures. Some results concerning frequencies are shown in Figure 1.

One major difference between the Greek verb lists and the lists from the other languages was the fact that the Greek lists contained significantly fewer words per list than the other lists, all $p_{\mathrm{s}}<.001$. The mean number of words per list for English was 37, Swedish 41, Polish 33 and Greek 18. 
For the Greek informants, as for the other language groups, the more frequently a verb occurred on the lists, the earlier it also appeared, Pearson's $r=-.48, p<.05$. This suggests that highly frequent verbs are easily accessed and therefore are produced early on during the task.

There was also a clear tendency to list basic motion verbs and verbs referring to basic human behaviours ("eat", "talk" etc. - see Table 1). The correlation between the four languages regarding listed verbs was significant for all pairwise language comparisons, all $p_{\mathrm{s}}<.005$, except for Polish and Greek. In this last case, the Pearson correlation coefficient was .26, $p>.20$.

Table 1.10 most frequently listed actions in the four languages. Verbs in bold face were found among the top ten entries in all four languages; verbs found in three of the four languages are marked by italics.

\begin{tabular}{|c|c|c|c|}
\hline Am. English & Greek & Polish & Swedish \\
\hline run & 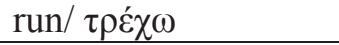 & write/ pisać & run /springa \\
\hline walk & walk/ $\pi \varepsilon \rho \pi \alpha \tau \alpha \dot{\alpha} \omega$ & jump/skakać & jump/hoppa \\
\hline jump & 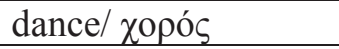 & read/ czytać & write /skriva \\
\hline swim & swim/ $\kappa о \lambda v ́ \mu \pi \imath$ & run/ biegać, biec & walk/gå \\
\hline talk/speak & write/ $\gamma \rho \alpha ́ \varphi \omega$ & eat/ jeść & eat/äta \\
\hline write & sleep/ v́ $\pi v o \varsigma$ & drink/ pić & swim/simma \\
\hline sleep & comb/ $\chi \tau \varepsilon \dot{\varepsilon} \nu 1 \sigma \mu \alpha$ & talk/mówić & laugh/skratta \\
\hline eat & eat/ $\mu \alpha \sigma o v \lambda \alpha \dot{\alpha} \omega$ & walk/ chodzić, iść & cry/gråta \\
\hline dance & 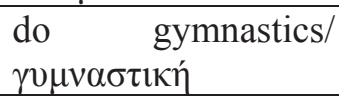 & sing/ śpiewać & talk/speak \\
\hline cry & $\begin{array}{l}\text { jump/ } \pi \eta \delta \dot{\omega} \\
\text { paint/ } \mu \pi \delta \gamma 1 \alpha \tau^{\prime} \zeta \omega\end{array}$ & $\begin{array}{l}\text { wash (oneself)/ } \\
\text { myć (się) }\end{array}$ & kiss/kyssas \\
\hline
\end{tabular}

Twenty-five of the most frequent verbs from the Greek lists were used to create a semantic space by using multidimensional scaling. Figure 2 shows the 3-dimensional solution for these verbs. As can be seen there is some tendency for vocal and mouth related actions to be close to one another. Verbs of bodily movement are also close to one another.

Correlation analyses were performed on the Euclidean distances between all 300 verb pairs for all 4 languages. With the exception of one correlation, the other 5 correlations were, although significant, ranged from $r=.18$ to .37. A larger correlation was obtained for the comparison between Swedish and Polish, $r=.56, p<.001$. This indicates that the network of semantic associations is similar for Swedish and Polish given the verbs included in the analysis. Noteworthy however is the finding that the semantic space for the Greek data did correlate with any of the other three languages. 


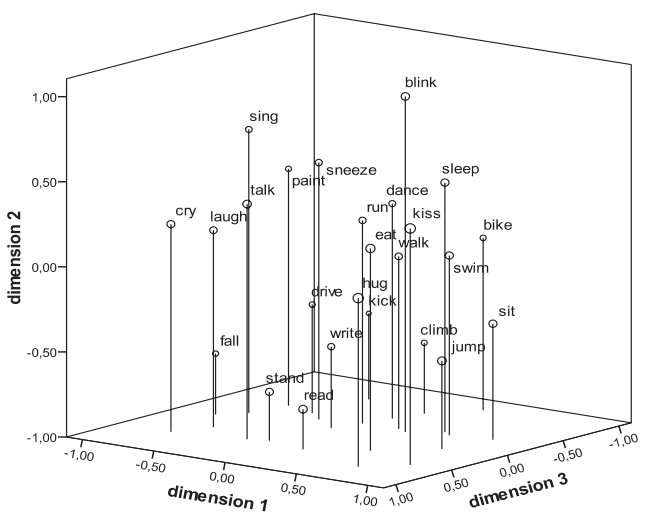

Figure 2. Euclidean distances between the Greek action verbs.

\section{Conclusions}

The limited number of words listed by the Greek informants is a factor that reduces the possibility of drawing reliable conclusions regarding the difference in lexical verb organisation between path and manner languages. One clear difference between the Greek data and the data from the three manner languages is that the correlation between semantic associations between Greek and the three manner languages appears to be relatively weak. This means that although similar actions are frequently listed by informants in the 4 languages, the associations between the verbs seem to be different. Nevertheless, the high frequency of motion and sound production verbs indicates that the cognitive status of natural human motion patterns and human vocal and emotional behaviour corresponds to similar lexical accessibility in all four languages.

\section{References}

Giese, M.A. and Poggio, T. 2003. Neural mechanisms for the recognition of biological movements. Nature Review Neuroscience 4, 179-192.

Hemeren, P.E. 1996. Frequency, ordinal position and semantic distance as measures of cross-cultural stability and hierarchies for action verbs. Acta Psychologica 91, 39-66.

Hemeren, P.E. and Gawronska, B. 2007 Lexicalization of natural actions and crosslinguistic stability. In Ahlsén, E. et al. (eds) 2007, Communication - Action Meaning. A Festschrift to Jens Allwood, 57-74. Göteborg, Sweden, Göteborg University.

Vinson, D.P. and Vigliocco, G. 2002. A semantic analysis of grammatical class impairments: semantic representations of object nouns, action nouns, and action verbs. Journal of Neurolinguistics 15, 317-351. 